5. - Quotient rings of rings with zero singular ideal, Pacific J. Math. 11 (1961), 1385-1392.

6. - Rings with zero right and left singular ideals, Trans. Amer. Math. Soc. 118 (1965), 150-157.

7. - Potent rings, Trans. Amer. Math. Soc. 119(1965), 524-534.

8. N. Jacobson, Structure of rings, Amer. Math. Soc. Colloq. Publ. Vol. 37, Amer. Math. Soc., Providence, R. I., 1956.

UNIVERSITY OF ROCHESTER

\title{
POWERS IN EIGHTH-GROUPS
}

\section{SEYMOUR LIPSCHUTZ}

1. Introduction. The purpose of this paper is to give an algorithm which decides whether or not an element in an eighth-group is a power. A group $G$ is an eighth-group if it is finitely presented in the form

$$
G=\operatorname{gp}\left(a_{1}, \cdots, a_{n} ; R_{1}\left(a_{\lambda}\right)=1, \cdots, R_{m}\left(a_{\lambda}\right)=1\right),
$$

where (i) each defining relator is cyclically reduced and (ii) if $B_{i}$ and $B_{j}$ are cyclic transforms of $R_{i}$ and $R_{j}$, then less than one-eighth of the length of the shorter one cancels in the product $B_{i}^{ \pm 1} B_{j}^{ \pm 1}$, unless the product is unity. The notation in this paper is the same as that in [3]. Note that Lemma 3 and Lemma 4 in [3] hold for eighth-groups.

Reinhart [4] gives an algorithm to decide, among other things, whether or not elements in certain Fuchsian groups are powers. Note that the Fuchsian group $F\left(p ; n_{1}, \cdots, n_{d} ; m\right)$, see Greenberg [1], is an eighth-group if

$$
4 p+d+m, n_{1}, \cdots, n_{d}>8 .
$$

Hence our algorithm holds for a somewhat wider class of groups and, furthermore, is purely algebraic.

REMARK. Given any word $V$ in a finitely presented group, it is possible to find a cyclically fully reduced word $V^{*}$ conjugate to $V$ by writing the word $V$ in a circle and then reducing. Such a word $V^{*}$ will be called a reduced cyclic transform of $V$.

2. The algorithm. First we prove a lemma about eighth-groups $G$. Here $r$ denotes the length of the largest defining relator in $G$.

Received by the editors April 3, 1964. 
LemMA. Let $W$ be cyclically fully reduced, let $W$ be conjugate to $V$, and let $l(V)=n$. Then $l(W) \leqq r^{2}+r n$.

Proof of Lemma. By Greendlinger's Basic Theorem in [2, p. 643], there exist reduced cyclic transforms $W^{*}$ and $V^{*}$ of $W$ and $V$ such that $W^{*}=T^{-1} V^{*} T$, where $l(T)<r / 8$ and $l\left(V^{*}\right) \leqq l(V)$. Hence

$$
l\left(T^{-1} V^{*} T\right)<r / 8+n+r / 8<r+n \text {. }
$$

Consequently, by Lemma 3 in [3],

$$
l\left(W^{*}\right) \leqq r l\left(T^{-1} V^{*} T\right) \leqq r^{2}+r n .
$$

But $W$ cyclically fully reduced implies $l(W)=l\left(W^{*}\right)$. Hence the lemma is true.

Suppose, now, that an arbitrary word $W \neq 1$ in an eighth-group is a power, say $W=V^{m}$ and $l(W)=n$. Let $A$ be a reduced cyclic transform of $V$; then $W$ is conjugate to $A^{m}$. Lemma 4 in [3] implies that $A^{m}=B$, where $B$ is cyclically fully reduced and where (i) $l(B) \geqq m$, and (ii) $l(B) \geqq l(A)-r$. Accordingly, our lemma above implies

$$
\begin{aligned}
m & \leqq l(B)<r^{2}+n r, \\
l(A) & \leqq l(B)+r<r^{2}+n r+r .
\end{aligned}
$$

The above discussion proves the following

THEOREM. Let $W \neq 1$ be an arbitrary word in an eighth-group $G$ where $l(W)=n$ and $r$ is the length of the largest defining relator in $G$. Then $W$ is a power if and only if $W$ is conjugate to $A^{m}$ where $m$ and $A$ satisfy (1) and (2).

Since the conjugacy problem has been solved for eighth-groups by Greendlinger in [2], and since there exist only a finite number of words in any given length, the above theorem gives us our algorithm.

\section{BIBLIOGRAPHY}

1. L. Greenberg, Discrete groups of motions, Canad. J. Math. 12 (1960), 415-426.

2. M. Greendlinger, On Dehn's algorithm for the conjugacy and word problems, with applications, Comm. Pure Appl. Math. 13 (1960), 641-677.

3. S. Lipschutz, An extension of Greendlinger's results on the word problem, Proc. Amer. Math. Soc. 15 (1964), 37-43.

4. B. Reinhart, Algorithms for Jordan curves on compact surfaces, Ann. of Math. (2) 75 (1962), 209-222.

Polytechnic Institute of BrookLyN 